\title{
User Acceptance of a Light-Emitting Diode Vest for Police Officer
}

\author{
Hyunjeong Han ${ }^{1)}$, Huiju Park ${ }^{2)}$ and Eunkyung Jeon ${ }^{1 \dagger}$ \\ ${ }^{1)}$ Dept. of Clothing \& Textiles, University of Ulsan; Ulsan, Korea \\ ${ }^{2)}$ Dept. of Fiber Science \& Apparel Design, Cornell University; Ithaca, USA
}

\begin{abstract}
This study aims to suggest practical considerations for designing protective clothing with increased visibility that will have higher user acceptance by law enforcement officers. Light-emitting diode(LED) patrol vests were visually and structurally assessed, and 125 police officers' responses from surveys about user acceptance of the vest were analyzed. The current LED patrol vest was designed for enhanced safety of police officers by increasing visibility in the dark. However, the user acceptance rate of the LED patrol vest indicates low use of and low satisfaction with the vest despite its enhanced safety features. In particular, differences in materials, design, functionality of the pockets and size of the vest depending on the hours worked, were statistically significant. The police officers' responses suggest areas of improvement in design, materials, ease of movement, size and functionality. Key issues include 'tactile discomfort'; 'impeded vision from the glare of the LED'; 'frequent malfunctions of the LED'; 'impossible repair of the broken LED units'; 'no user feedback'; 'inconvenient to replace batteries'; 'brittle materials' and 'unpleasing look'. To increase user acceptance, designer should incorporate context-awareness, a convenient user interface, a modular design approach, first responders' self-image as public servants in relation to their aesthetic perspectives of their uniforms, and scientific evaluation of the effectiveness of the intended functions of the clothing. Suggested implications for designing the LED patrol vest can be applied to designing other functional/protective clothing for intended end users with special needs.
\end{abstract}

Key words: LED patrol vest, user acceptance, protective clothing, police officer

\section{Introduction}

More than one million people around the globe die every year on roads, and over 20 million suffer road traffic injuries(World Health Organization, 2009). In Korea, where the registered units of cars are reaching 200 million ("Car", 2013), over 200,000 traffic accidents occur and over 5,000 people die of traffic accidents every year(Road Traffic Authority, 2012).

Low luminance and visibility at night dramatically increase the number of deaths and fatal injuries of people who are hit by vehicles on roads(Plainis, Murray and Pallikaris, 2006). In particular, first responders, such as law enforcement officers who work on roads, are vulnerable to the environmental challenge of working near moving vehicles. Kang(2009) reported that the number of traffic accidents was greater at night (after 10pm) while the visibility is low on the road. A review of literature reveals that dark, low-contrast clothing is a contributing factor to road traffic accidents at night because drivers' eyes respond to those garment conditions slowly, and this impedes quick reactions for stopping a vehicle in an emergency(Plainis, Murray and Pallikaris, 2006). For this reason, in many countries police officers who are engaged in a range

†Corresponding author; Eunkyung Jeon

Tel. +82-52-259-2842, Fax. +82-52-259-2888

E-mail: ekjeon@ulsan.ac.kr of traffic activities wear high-visibility vests or jackets with lightreflective trim.

To increase visibility on roads, reflective tape has been widely used not only on traffic law enforcement personnel's patrol vests but also on the garments for joggers, cyclists, construction workers, etc. because the reflective tape made of polyester or polyester blend with cotton is washable, sew-able and relatively inexpensive. However, it is almost impossible to increase visibility without an external light source reflecting on the tape(Kim, 2010).

Recently developed light emitting diode(LED) technology began to be incorporated into clothing as a safety feature because LEDs can provide increased visibility regardless of the environmental light conditions. LED is known as a relatively inexpensive technology that generates its maximum luminance faster and is more durable against impact shock than conventional light bulbs(Lee, 2008). As industries become more aware of its positive impacts of energy efficient high luminescent features on safety, the LED technology is used not only to improve safety, but also to provide value-added functionalities to conventional clothing(Gould, 2003). For example, Philips Inc. introduced emotional responsive clothing by embedding biosensors and LEDs. The clothing was designed to reflect the wearer's emotion based on characteristics of bio-signals collected in the wearer's different emotional states. In addition, the sportswear industry began to incorporate LEDs for 
increased visibility for runners, cyclists, joggers, and skiers who need visibility safety features(Gould, 2003).

Considering the LED's technological merits such as energy-efficient high luminance and durability, the use of LEDs is also expected to provide enhanced safety for police officers working on roads at night than conventional reflective trims do because of its capability of flashing lights. In terms of the effectiveness of increasing visibility, Wood et al.(2009), their experimental study, reported that flashing lights on cyclists showed slightly higher perception of visibility than attaching reflective trims both in daytime and at night.

Recently, in South Korea, patrol vests equipped with LEDs have been given to police officers for enhanced protection. The integration of LED technology is intended to enhance personal protection. For police officers and other first responders, the given uniform that they are wearing is, in many cases, the only, and irreplaceable protection. It functions as armor, a buffer, or sometimes an insulator from environmental hazards in officers' given work conditions, in which there are limited or no alternatives for protection. Improved designs with enhanced safety features in protective clothing can improve the safety and work efficiency of police officers. Therefore, it is imperative to assess the officers' acceptance of and requests regarding their protective clothing in order to understand their needs and to then be able to increase their safety and efficiency on the job. Considering the fact that police officers are typically required to wear items provided by their governing organizations through an official procurement process, it is important to reflect the wearers' needs in the design.

This study assesses the shape and the structure of current LED patrol vest, and traffic law enforcement officers' acceptance of a LED patrol vest used by the police in South Korea; in addition, it evaluates the suggestions that the officers in the study offered for improvement of the vest based on their experiences with it. The aim of this study is to suggest practical considerations for designing protective clothing with increased visibility and higher user accep- tance by law enforcement officers.

\section{Methodology}

\subsection{Analysis of LED patrol vests}

Researchers identified the LED patrol vests through on-site visits to police departments in the southern area of South Korea. The structure of the electric components for the LED operation and the design features were visually analyzed. The analysis was carried out in two steps. The researchers visited the police office and photographed and studied the image of a policeman wearing a LED patrol vest and the detailed image of the outside and the inside of the vest. Then, the researchers disassembled the LED vest and analyzed the detailed structure.

\subsection{Survey of user acceptance}

The initial survey questions were developed based on interviews with police officers and previous studies about user acceptance of protective apparel by $\operatorname{Kim}$ and $\operatorname{Suh}(2002,2003)$ and $\operatorname{Lim}$ et al.(2006, 2008). A pre-test was conducted with 50 police officers. After the pre-test, the wording of the questionnaire was revised to clarify the instructions and questions. The revised survey was used for data collection in South Korea; the researchers visited a police training institute and conducted the survey with the permission of the organization. Participation in the survey was voluntary.

The first part of the survey contained three general questions about the respondents: age, duration of work as a traffic police officer, and average daily work hours on roads. The next part asked about the respondents' acceptance of the LED patrol vest, including their experience and satisfaction with the LED vest, their reasons for their dissatisfaction, and their needs for improved comfort and design for the vest, as shown in Table 1. All questions were multiple choices, but open-ended comments were included to ensure that the participants' responses were not limited to the choices given by the researchers. Out of 250 surveys, only 125

Table 1. Survey questions

\begin{tabular}{cl}
\hline Categories & Qge \\
\hline Information about participants & $\begin{array}{l}\text { Duration of work as a traffic police officer } \\
\text { Average daily work hours on the road }\end{array}$ \\
\hline Usage and acceptance & Experience wearing LED patrol vest \\
& Frequency of usage of LED function \\
& Reasons for less/no use of the LED function \\
& Satisfaction of the LED vest \\
& Reasons for dissatisfaction and needs for improvement \\
& Perception of contribution of the LED function to participants' safety \\
\hline
\end{tabular}


responses were analyzed, collected from police officers who had experience wearing the LED patrol vest.

\subsection{Data analysis}

The data were analyzed using multiple response analysis. Oneway ANOVA and multiple comparison tests were also used to identify a significant relationship between the participants' work hours and satisfaction with the vest using PASW Statistics 18.0. Partici- pants' open-ended comments were also included in the analysis.

\section{Results}

\subsection{Analysis of LED patrol vests}

In this study, the LED patrol vest that the police officers wore has three wide light-reflective tapes attached to the front and back of the vest, which was made of mesh, as shown in Table 2.

Table 2. LED patrol vests for traffic police officers

Whole Image Interior

Controller

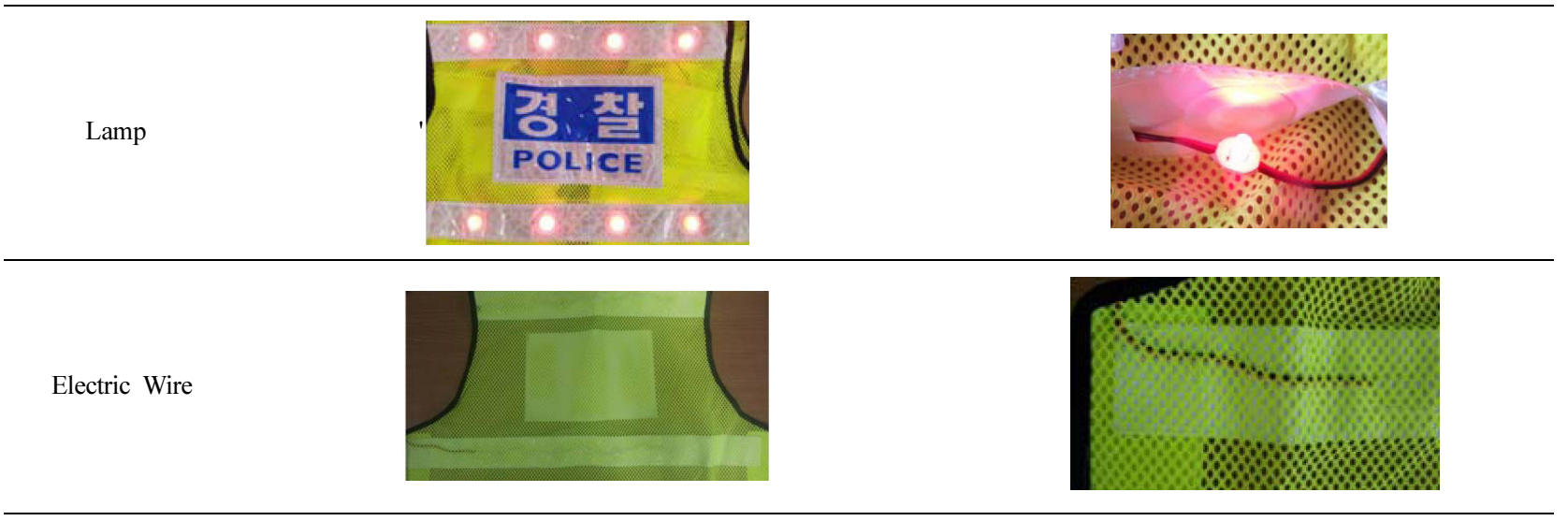

Battery


A total of eight LEDs are located on the back of the vest; four LEDs are placed on each line of the light reflective tape around the shoulder and the mid-back. The placement of LEDs only on the back is expected to increase battery consumption because if the police officers cannot see whether the LEDs are on or off, they are less likely to pay attention to the need to turn them on and off, depending on the environmental light conditions. Each LED (diameter: $0.4 \mathrm{~cm}$, height: $0.6 \mathrm{~cm}$ ) emits a red light and is covered by a hemisphere-shaped plastic capsule (diameter: $2 \mathrm{~cm}$, height: $0.8 \mathrm{~cm}$ ) to reduce glare from the LED. The capsule also protects the LEDs from ambient humidity and damaging physical contact. The LED function is operated by a toggle switch; pressing the switch once turns on the LEDs to flash at one second intervals, and pressing the switch once more turns them off. Two 1.5 voltage AA batteries are connected to the LEDs and an on/off controller(dimension: $3 \mathrm{~cm} \times 2 \mathrm{~cm} \times 0.5 \mathrm{~cm}$ ) through wires. The two batteries are located inside a patch pocket (dimension: $9 \mathrm{~cm} \times 10 \mathrm{~cm}$ ) and the wearers are encouraged to replace the old batteries with new ones when needed. The batteries can cause inconvenience for wearers because they are little heavy and impossible to reuse. Therefore, light-weighted and rechargeable batteries could be a better option.

\subsection{Information about participants}

All the respondents were male police officers, and a majority of them were in their $30 \mathrm{~s}(42.4 \%)$ and $40 \mathrm{~s}(44.8 \%)$, as shown in Table $473.6 \%$ of the respondents had less than five years of work experience as traffic police $82.4 \%$ of the respondents reported that they work more than five hours a day on roads (Table 2), $48 \%$ of the respondents reported 5-10 hours of work a day, and $34.4 \%$ more than 10 hours.

\subsection{User acceptance of the LED patrol vest}

Experience of wearing LED vests: $77.6 \%$ of the survey participants reported that they used the LED function(Table 4): $54.4 \%$ of

Table 3. Schematic diagrams of LED patrol vests

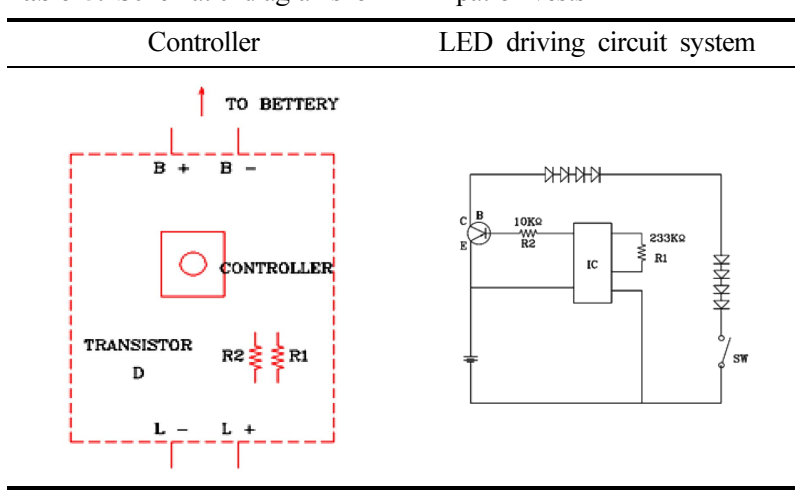

Table 4. Information about respondents

\begin{tabular}{|c|c|c|}
\hline \multicolumn{2}{|c|}{ Factors } & Number of responses $(\%)$ \\
\hline \multicolumn{2}{|r|}{$20 \mathrm{~s}$} & $8(6.4)$ \\
\hline \multicolumn{2}{|r|}{$30 \mathrm{~s}$} & $53(42.4)$ \\
\hline \multirow[t]{3}{*}{ Age } & $40 \mathrm{~s}$ & $56(44.8)$ \\
\hline & Over 50 & $8(6.4)$ \\
\hline & Total & $125(100)$ \\
\hline \multirow{5}{*}{$\begin{array}{l}\text { Work duration } \\
\text { as a traffic } \\
\text { police officer }\end{array}$} & 5 years & $92(73.6)$ \\
\hline & $5 \sim 10$ years & $25(20.0)$ \\
\hline & $10 \sim 15$ years & $7(5.6)$ \\
\hline & Over 15 years & $1(0.8)$ \\
\hline & Total & $125(100)$ \\
\hline \multirow{4}{*}{$\begin{array}{c}\text { Average daily } \\
\text { work hours road } \\
\text { on the road }\end{array}$} & Less than 5 hours & $22(17.6)$ \\
\hline & $5 \sim 10$ hours & $60(48.0)$ \\
\hline & $10 \sim 15$ hours & $43(34.4)$ \\
\hline & Total & $125(100)$ \\
\hline
\end{tabular}

the participants used it occasionally, while only $23.2 \%$ used it all the time. However, $22.4 \%$ of the participants reported that they never used it. There was no statistically significant difference in the usage of the LED function among three different work hour groups.

Reasons for no/less use of the LED function: This includes "frequent malfunction of $\operatorname{LED}(51.0 \%)$ "; "habitually didn't use (16.9\%)"; "felt it was not necessary(13.5\%)"; and "inconvenient to use(10.2\%)"; as shown in Fig. 2. In particular, the inconvenient location of the switch, frequent battery changes, and not being able to see the LEDs on the back of the vest were given as detailed reasons for the inconvenience of use. Other reasons (8.5\%) included "need to purchase batteries"; and "uncomfortable feeling of wearing a flashing LED vest."

Satisfaction with the current LED patrol vest: Satisfaction with the vest was assessed using a 5-point Likert-type scale in terms of design, materials, ease of movement, functionality of pockets, and the size of the vest, as shown in Table 5. The longer the police officers worked, the less satisfied they tended to be with the vest

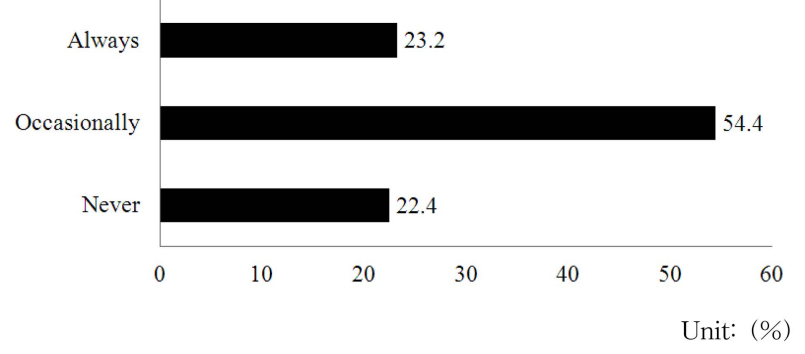

Fig. 1. LED functions utilization by work hours on roads. 


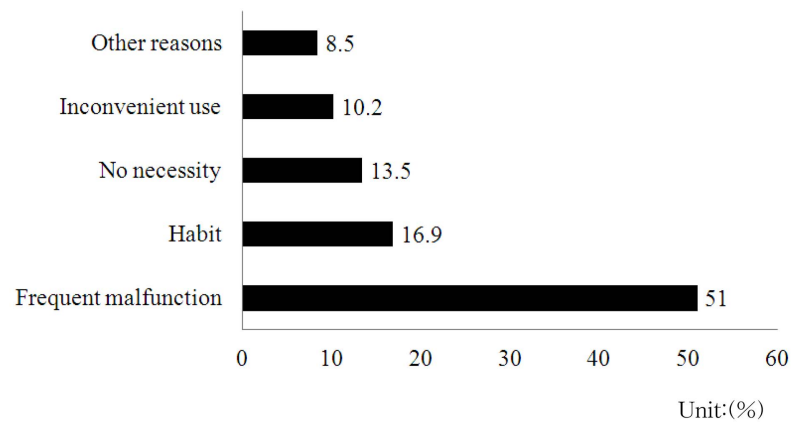

Fig. 2. Reasons for no/less use of the LED function.

Table 5. Results of one-way ANOVA regarding the participants' satisfaction with the vest

\begin{tabular}{|c|c|c|c|c|}
\hline & & Work hours & & \\
\hline & $<5$ hours & 5-10 hours & $10-15$ hours & F-value \\
\hline & Mean S.D. & Mean S.D. & Mean S.D. & \\
\hline Decion & $2.82 \quad .808$ & $2.84 \quad .958$ & $2.10 \quad .700$ & $6153 * * *$ \\
\hline Design & $\mathrm{a}$ & $\mathrm{a}$ & $\mathrm{b}$ & 0.153 \\
\hline Materials & $2.76 \quad .902$ & $\begin{array}{ll}2.68 & .752\end{array}$ & $\begin{array}{ll}1.81 \quad .750\end{array}$ & $11167 * * *$ \\
\hline IVialentals & $\mathrm{a}$ & $\mathrm{a}$ & $\mathrm{b}$ & $11.10 / 7=$ \\
\hline $\begin{array}{l}\text { Ease of } \\
\text { movement }\end{array}$ & $2.64 \quad .895$ & $2.77 \quad .865$ & $2.29 \quad .845$ & 2.571 \\
\hline Functionality & $\begin{array}{ll}.03 \quad .918\end{array}$ & $\begin{array}{ll}.03 \quad .925 \\
\end{array}$ & $\begin{array}{ll}2.33 & .796\end{array}$ & \\
\hline of pockets & $\mathrm{a}$ & $\mathrm{a}$ & b & $5.177 * *$ \\
\hline Siz & $2.61 \quad .966$ & $2.69 \quad .855$ & $\begin{array}{ll}2.14 & .854\end{array}$ & $312+2$ \\
\hline & $\mathrm{a}$ & $\mathrm{a}$ & b & 0.122 \\
\hline
\end{tabular}

*** $p<.001,{ }^{* *} p<.01,{ }^{*} p<.05$

$\mathrm{ab}(\mathrm{a}>\mathrm{b})$ means with a common letter in the same row are not significantly different (Duncan, $\alpha=.05$ )

regarding the five factors asked about in the survey. In particular, differences in materials, design, functionality of the pockets and size depending on the hours worked, were statistically significant. In all the factors the police officers' response showed low satisfaction level(below 3 points on average in 5 point Likert scale), which implies a need for an further understanding of reasons for dissatisfaction for improvement in user acceptance.

A total of 85 open-ended responses indicate specific reasons for dissatisfaction with the vest and further needs, shown as follows.

Design: Every working hour group of police officers expressed low level of satisfaction below 3.0 with Design of LED patrol vest. A significant difference in satisfaction with Design of LED patrol vest was found among the three different groups by work duration as shown in Table 3. Issues that were addressed include bulkiness of LED bulbs; inconvenient location of the LEDs and switch; inappropriate design of user interface; and lack of understanding of con- text awareness. These issues appear to directly cause tactile discomfort, impeded vision due to glare, inconvenience of usage in a certain context, and lack of durability. For example, some participants reported that bulky protruding ball-shaped LED covers cause uncomfortable pressure while participants were seated in a vehicle and leaning on something. The participants also reported that they don't even know whether the LEDs are turned on or not because they can't see them located on their backs. This indicates inappropriate design of user interface. In addition, the participants were often hesitant to turn the LEDs on because the LEDs impede their nearby colleagues' vision. The participants also reported frequent malfunctions of the switch. Some police officers reported they want a more aesthetically pleasing or at least acceptable design.

Materials: Statistical analysis reveals that longer work duration led to lower satisfaction as shown in Table 3. Some reported that the reflective tapes were too stiff and brittle. So they broke easily, especially during winter. The broken parts of the tapes have sharp edges which can hurt the wearers. They strongly demanded for a material that has more comfortable wearing sensation. This result was consistent the previous study about police officers' perception about their multi-functional safety vest(Han \& Jeon, 2012).

Ease of movement: Respondents showed low satisfaction in all groups regardless of working hours. Some reported that the armholes are not big enough for comfortable arm movement when the officers are wearing their winter clothing.

Functionality of pockets: ANOVA result showed significant difference according to working hours $(\mathrm{p}<.01)$, and groups that had longer working hours appeared to have great dissatisfaction in the function of the pockets. Some reported that the hook and loop used as a closure system for the pockets is not durable, and police officers often drop items on the ground due to the unreliable fasteners. In addition to the ratings and comments about the five suggested design features, participants' open-ended comments described inconvenience in maintenance for both electric and textile components; the participants reported that they have to buy extra batteries on their own to continue using the LED functions because the vest comes with only one non-rechargeable battery. In addition, they can't easily replace or repair the broken LEDs electric units because police officers cannot detach the electric components, which are all non-separable.

Size of the vest: Low level of satisfaction was shown in all groups with statistically significant difference $(\mathrm{p}<.05)$ between working hour groups. Reported responses in this study and previous study(Han \& Jeon, 2012) show that vests are often shared by multiple officers in each police department. Therefore, typically big sizes are offered, which causes an unpleasing appearance for smaller figures, especially during summer. 
Table 6. Perceived contribution of LED to the safety on roads unit:\%

\begin{tabular}{ccccc}
\hline & $<5$ hours & $5-10$ hours & $10-15$ hours & Total \\
\hline Very little & 0 & 3.4 & 0 & 3.4 \\
Little & 0 & 6.0 & 1.7 & 7.8 \\
Neutral & 12.1 & 20.7 & 6.0 & 38.8 \\
Large & 10.3 & 22.4 & 6.0 & 38.8 \\
Very large & 2.6 & 5.2 & 3.4 & 11.2 \\
Total & 25.0 & 57.8 & 17.2 & 100 \\
\hline Mean & 3.62 & 3.34 & 3.65 & 3.47 \\
S.D. & .677 & .993 & .933 & 0.918 \\
\hline
\end{tabular}

F-value: 1.432, P: .245

Perception of contribution of the LED vest to safety: $50 \%$ of the respondents reported that they believe the LED function had a positive effect on increased visibility and their safety as shown in Table $4 ; 38.8 \%$ of the respondents believed that the contribution of the LED function to their safety was 'large' and $11.2 \%$ of them said 'very large'. However, $11.2 \%$ of the respondents reported that the contribution of the LED function to their safety was 'little(7.8\%)' or 'very little'(3.4\%). This negative response seems to be related to the aforementioned reasons for less/no use(Fig. 2) such as frequent malfunction and inconvenient use of the LED. There was no significant difference between the groups shown in the recognition on LED contribution according to working hours.

\section{Conclusions}

In this study, the visual shape and the structure of the LED patrol vest currently worn by traffic police officers were assessed, and usages and acceptances on LED patrol vests were investigated from actual users. Also, the problems of LED patrol vests and the demands were investigated through the survey and open-ended responsesto give useful information for patrol uniform design for improved safety and comfort for police officers.

Visual and technical assessment of LED patrol vest identified the enhanced safety features, which may be helpful to reduce injuries and accidents of police working in the dark by increasing their visibility; The current police LED patrol vests were constructed with 3 layers of luminous reflective tapes in front and back, 2 layers of 8 LED lamps on the back, and the entire neon-colored net for enhanced visibility. However, some technical problems were found such as needs for battery replacement or recharging, and unawareness of on/off of the LED. Despite the respondents' high acknowledgement of enhanced safety feature, the findings of this study indicate the user acceptance of LED patrol vest was low. This implies that the police officers who participated had needs for improvements in the design of the vest.
A statistical analysis found a significant correlation between hours of duty and satisfaction; an increase in working hours resulted in significantly lower satisfaction with design, materials, functionality of pockets and the size of the vest. This result implies that increased user experience leads to lower satisfaction with the vest, indicating the needs for improved design of the LED patrol vest.

The current study further identified areas of improvement through survey and open-ended questions.

Key design issues for improved user acceptance encompass design, ease of movement, materials, functionality of pockets and size of the vest, as shown in following keywords: "tactile discomfort due to bulky LED protection cap"; "impeded vision from the glare of the LEDs"; "inability to repair or replace the malfunctioning LEDs due to non-separable design of electric unit"; "frequent malfunctions of LEDs"; "no information about LED's on/off status due to inconvenient location of LEDs"; "inconvenience of replacing batteries frequently"; "Brittle materials"; and "unpleasing look".

These findings provide practical implications for designing improved vests for police officers. First, designers need to understand the intended end-users' (police officers in this study) work environments, required activities for law enforcement on roads, and context to improve user acceptance of the design. A convenient user interface should also be considered to make the wearer aware of the operation of the intended functions. A modular design approach should be taken for convenient maintenance. Easily detachable design features will allow for easy repair and replacement of broken electrical parts. The findings of this study also show a discrepancy between police officers' endorsement of enhanced visibility aids and their actual use, which was consistent with results of previous studies(Hagel et al., 2007; Wood et al., 2007). Therefore, this study suggests that police officers should be educated about the importance of having visibility aids.

In designing the LED vest or even other garments for police officers, designers need to take into serious consideration the police officers' self-image as law enforcement personnel working in public spaces. In this study, respondents showed their low satisfaction with the appearance of "light-flashing garment". This may contribute to a low rate of use of the LED patrol vest among police officers even though they may understand that LED functions can provide increased visibility on the road. Last, scientific evaluation of design effectiveness on increased visibility and decreased accidents in comparison to existing light-reflective patrol vests without LED functions needs to be done. Those future studies are expected to provide a clear understanding of the effectiveness of LED functions, which may help police officers decide whether to use them or not.

This study focused on an analysis of user acceptance of one of the commonly used current LED patrol vests and suggested prac- 
tical implications for improvement of the design to increase user acceptance. Although the study investigated only one product, the implications of this study may be applied to designing other functional clothing for intended end-users with special needs.

\section{Acknowledgement}

This research was supported by the 2012 Research Fund of University of Ulsan.

\section{References}

'All traffic accidents(Police DB)'. (2012, December 31). Road traffic authority. Retrieved August 10, 2013, from http://taas.koroad. or.kr/reportSearch.sv?s_flag $=02 \#$

'Car-The number of registration vehicle 20million is just before'. (2013, July 11). Ministry of land, infrastructure, and transport. Retrieved August 10, 2013, from http://korealand.tistory.com/1772

Global status report on road safety: time for action. (2009). Geneva, World Health Organization. Retrieved May 12, 2013, from http:// www.who.int/violence_injury_prevention/road_safety_status/2009).

Gould, P. (2003, October). Textiles gain Intelligence. Materials Today, pp. $38-43$.

Hagel, B. E., Lamy, A., Rizkallah, J. W., Belton, K. L., Jhangri, G. S., \& Cherry, N. (2007). The prevalence and reliability of visibility aid and other risk factor data for uninjured cyclists and pedestrians in Edmonton, Alberta, Canada. Accident Analysis \& Prevention, 39, 284-289.

Han, H. J., \& Jeon, E. K. (2012). An analysis of the demands for the improvement of versatile safety vests for traffic police officers. Journal of the Korean Society for Clothing Industry, 14(1), 100-108.

Kang, M. J. (2009). Traffic accident analysis and traffic police activity - Centering Gwangju province police agency. Journal of Korea Contents Association, 9(9), 681-691.

Kim, H. R., \& Suh, M. A. (2002). A research of worker`s uniform in the mechanical industry. The Research Journal of the Costume Culture, 10(6), 718-734.

Kim, H. R., \& Suh, M. A. (2003). A study on satisfaction of worker's uniform in the mechanical industry. The Research Journal of the Costume Culture, 11(6), 867-878.

Kim, M. H. (2010). A study for developing the prototype of LED safety vest. The Research Journal of the Costume Culture, 18(3), 488-498.

Lee, H. K. (2008). A study on the design of smart photonic clothing based on inorganic EL technology. Unpublished master's thesis, Yonsei University, Seoul, Korea.

Lim, H. J., Choi, H. S., Lee, K. M., \& Kim, S. A. (2006). A study on the actual wearing of conditions working uniform for the aircraft mechanic. Journal of the Korean Society of Clothing and Textiles, 30(9), 1344-1353.

Lim, H. J., Choi, H. S., Lee, K. M., \& Kim, S. A. (2008). Ergonomic design of working uniform for the aircraft mechanics. Journal of the Korean Society of Clothing and Textiles, 32(5), 681-691.

Plainis, S., Murray, I. J., \& Pallikaris, I. G. (2006). Road traffic casualties: Understanding the night time death toll. Injury Prevention, 12(2), 125-128.

Wood, J. M., Lacherez, P. F., Marszalek, R. P., \& King, M. J. (2009). Drivers' and cyclists' experiences of sharing the road: incidents, attitudes and perceptions of visibility. Accident Analysis \& Prevention, 41(4), 772-776.

(Received 6 August 2013; 1st Revised 12 August 2013; 2nd Revised 1 October 2013; 3rd Revised 4 October 2013; Accepted 13 October 2013)

Copyright (C) The Society of Fashion and Textile Industry. 2013. This is an open access article distributed under the terms and conditions of the Creative Commons Attribution Non-Commercial license (http://creativecommons.org/licenses/by-nc/3.0/), which permits unrestricted non-commercial use, distribution, and reproduction in any medium, provided the original work is properly cited. 